\title{
Spikes with short inter-spike intervals in frog retinal ganglion cells are more correlated with their adjacent neurons' activities
}

\author{
Wen-Zhong Liu, Ru-Jia Yan, Wei Jing, Hai-Qing Gong, Pei-Ji Liang ${ }^{\bowtie}$ \\ School of Biomedical Engineering, Shanghai Jiao Tong University, Shanghai 200240, China \\ Correspondence: pjliang@sjtu.edu.cn \\ Received July 13, 2011 Accepted August 28, 2011
}

\begin{abstract}
Correlated firings among neurons have been extensively investigated; however, previous studies on retinal ganglion cell (RGC) population activities were mainly based on analyzing the correlated activities between the entire spike trains. In the present study, the correlation properties were explored based on burst-like activities and solitary spikes separately. The results indicate that: (1) burst-like activities were more correlated with other neurons' activities; (2) burst-like spikes correlated with their neighboring neurons represented a smaller receptive field than that of correlated solitary spikes. These results suggest that correlated burst-like spikes should be more efficient in signal transmission, and could encode more detailed spatial information.
\end{abstract}

KEYWORDS correlated firing, burst-like spikes, solitary spikes, correlation index, receptive field

\section{INTRODUCTION}

All visual information sent to the central nervous system is transmitted through spike activities of retinal ganglion cells (RGCs). Previous studies have shown that not every retinal spike fired by RGC can evoke action potential in its postsynaptic target neuron(s) (Usrey et al., 1999; Sincich et al., 2007), and that retinal spikes with a short inter-spike interval (ISI) (generally $\leqslant 30 \mathrm{~ms}$ ) are more efficient to drive corresponding lateral geniculate neurons to fire (Hirsch et al., 1998; Rowe and Fischer, 2001; Sincich et al., 2007). On the other hand, correlated firing activities were observed among RGCs (Brivanlou et al., 1998; Schneidman et al., 2006), and it was suggested that RGCs might encode visual information in a dynamic population way (Schnitzer and Meister, 2003; Field and Chichilnisky, 2007). Although correlated activities among RGCs have been extensively studied, previous studies on RGC population activities were mainly based on the entire spike trains. Moreover, firing activities within a spike train are not evenly distributed, and a recent spiking history usually has effective influences on the cell's successive firing activities (Pillow et al., 2008; Liu et al., 2011). In the present study, we explored the correlated firings of single neuron's activities with different ISIs, and examined the physiological significance of spikes with a short ISI.

Population RGCs' activities in response to pseudorandom checker-board flickering were recorded via multi-electrode arrays (MEA). Spike activities were classified into two categories: (1) burst-like spikes (with $|S|<30 \mathrm{~ms}$ ) and (2) solitary spikes (with $|S| \geqslant 30 \mathrm{~ms}$ ). For both parts, correlation index $(\mathrm{Cl})$, which indicates the degree of one cell's firing activity in correlation with its neighbor neurons, was calculated for each individual cell being investigated. It was found that, for a single spike train, those spikes with a short ISI were with a higher probability being correlated with its adjacent neurons as compared to the solitary spikes. In addition, burstlike spikes correlated with other cells represent a smaller receptive field as compared to correlated solitary spikes, suggesting that burst-like spikes encode more detailed visual information.

\section{RESULTS}

Correlation indices calculated based on burst-like spikes and solitary spikes

As reported previously, correlation strength between two neurons is negatively related to the distance between them, 
and correlation strength is very weak when the distance is bigger than $300 \mu \mathrm{m}$ (Liu et al., 2011). So in this paper, we only consider correlation activities between two neurons with their distance less than $300 \mu \mathrm{m}$.

Spike activities in each train were classified into two categories: burst-like spikes and solitary spikes (Fig. 1). Raster plot shows that within a single spike train, the burst-like spikes were more likely to be correlated with the spikes fired by its adjacent neurons (Fig. 2). To examine the correlated firing properties related to these two kinds of spike activities quantitatively, correlation indices were calculated for both parts separately. Results of 48 neurons from 3 retinas are plotted in Fig. 3A, which shows that burst-like spikes in a spike train were more likely to be correlated with other neurons' firing activities as compared to solitary spikes (burstlike: $0.22 \pm 0.14$, solitary spikes: $0.17 \pm 0.11, p<0.05$ ) (Fig. 3B).

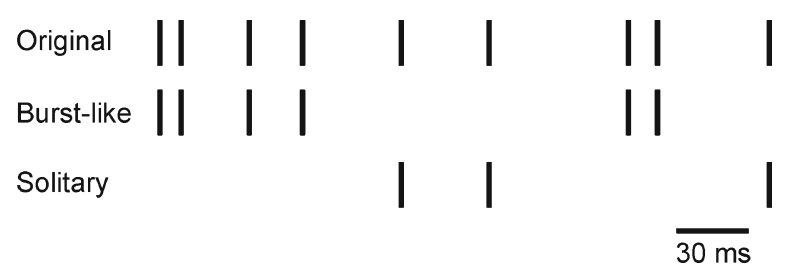

Figure 1. Separating an original spike train into two subsequences. Burst-like activities, spikes with $|S|<30 \mathrm{~ms}$ in the original sequence; solitary action potentials, spikes with ISI $\geqslant 30 \mathrm{~ms}$ in the original sequence.

\section{Cross-correlogram analysis based on burst-like and solitary firing activities}

The $\mathrm{Cl}$ provides general statistical results that burst-like spikes are more likely to be correlated with other neurons' activities as compared to the solitary ones. To further look into the details of the correlated firing structure of these two different parts, cross-correlogram analysis was applied (Fig.4). The Raster plot in Fig. 4A shows part of two spike trains; Fig. 4B (left panel) illustrates the cross-correlogram of the two firing sequences $A$ and $B$; cross-correlograms calculated based on solitary and burst-like spikes are also displayed (middle and right panels). The cross-correlogram calculated using burst-like spikes has a larger central peak value as compared to that calculated using solitary spikes, which suggests that the firing activities were more correlated with its neighboring RGCs when the neuron fired burst activities. Besides, the cross-correlogram based on burst-like spikes has bigger side peaks at a time lag of 5-10 ms, which may suggest stronger common input from an amacrine cell to the couple of ganglion cells via gap junction (Brivanlou et al., 1998).

Considering that the central/side peaks are both within $\pm 10 \mathrm{~ms}$ in the cross-correlogram, while the $\mathrm{Cl}$ values were

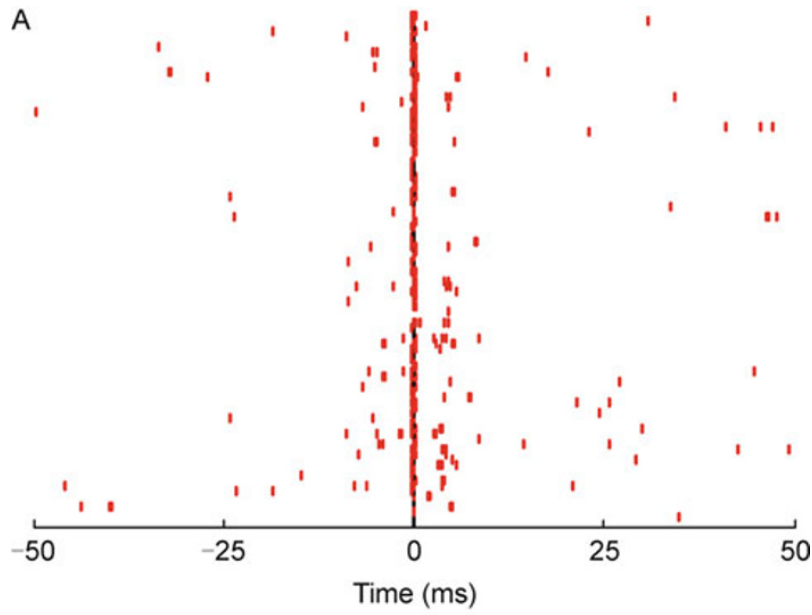

B

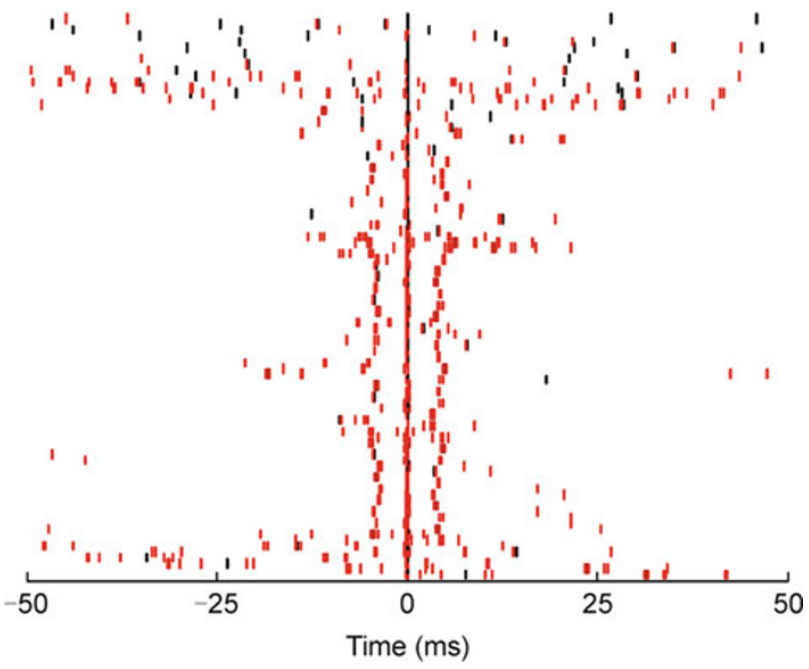

Figure 2. Relationship between spikes fired by the examined neuron and that from its neighboring neurons (within an inter-cellular distance of $\mathbf{3 0 0} \boldsymbol{\mu m}$ ). (A) A set of 100 solitary spikes from a spike train (black dot: spikes from the cell being examined; red dot: spikes from its neighboring neurons). (B) A set of 100 burst-like spikes from the same spike train. Raster were centered at $\mathrm{t}=0$ either for the solitary spikes or burst-like spikes of the examined spike train.

estimated with a resolution of $20 \mathrm{~ms}$ in our analysis, bigger central/side peaks in cross-correlogram for burst-like spikes may account for the bigger $\mathrm{Cl}$ values. Results plotted in Fig. 5 clearly show that burst-like spikes have bigger side peak values as well as central peak values.

\section{Receptive field represented by correlated firings}

It was previously reported that correlated firings are related to better spatial resolution (Meister et al., 1995; Schnitzer and Meister, 2003; Li et al., 2011). Given that burst-like spikes were more correlated with the neighboring neurons' activities 


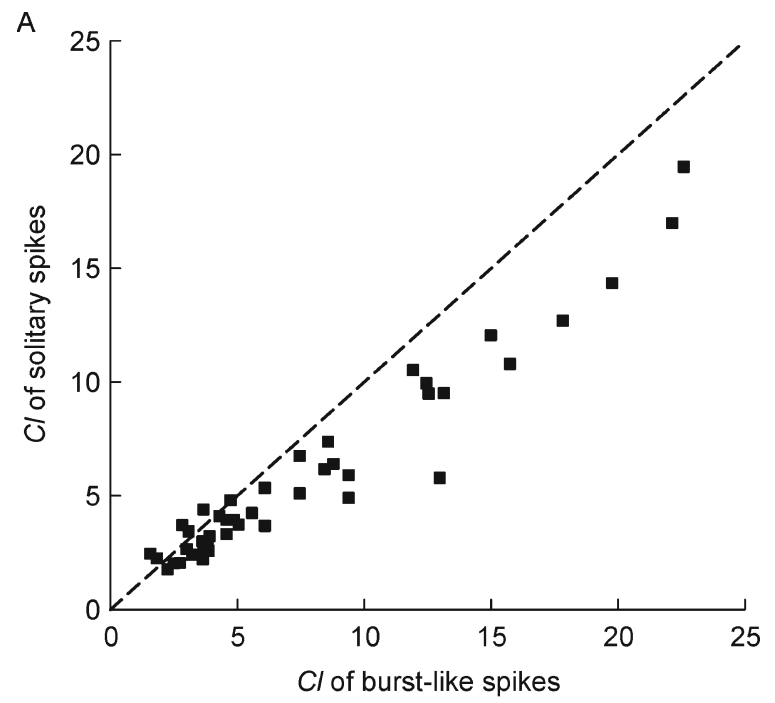

B

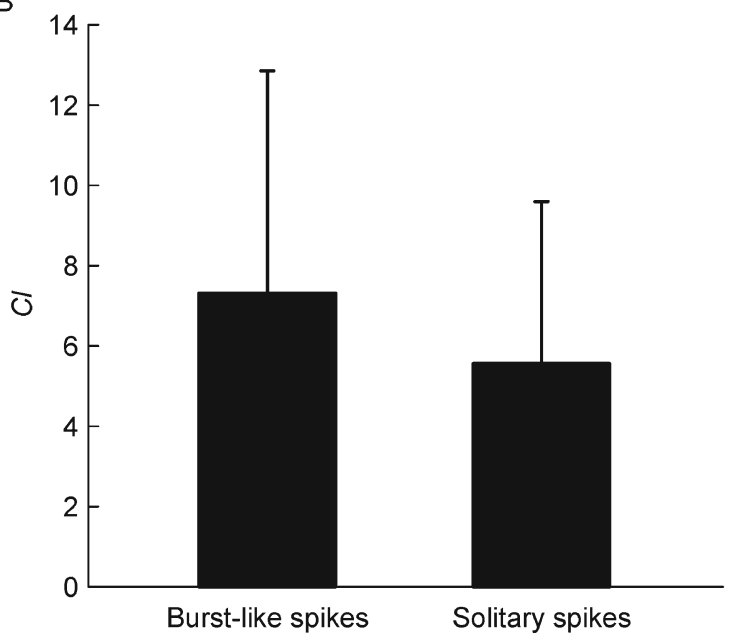

Figure 3. The comparison of correlation index (Cl) calculated based on burst-like and solitary-spike sub-sequences. (A) Scatter diagram of the comparison. Each dot represents the calculation result for a single neuron; (B) Statistics results (mean \pm SD, burst-like: $0.22 \pm 0.14$, solitary spikes: $0.17 \pm 0.11$, paired $t$ test, $n=48, p<0.05$ ).

as compared to solitary spikes, we further examined whether or not a neuron's burst-like spikes being correlated with its neighboring neuron's activity represent smaller receptive fields. Totally 16 neurons from 3 retinas were investigated. Our results show that for each individual cell, the receptive field area estimated using burst-like spikes being correlated with one of its adjacent cells represents smaller receptive field center as compared to that estimated using all the burst-like spikes (Fig. 6A); correlated solitary spikes also represent smaller receptive fields than the whole solitary spikes sequence (Fig. 6B). Furthermore, as plotted in Fig. 6C burst-like spikes being correlated with other cells also represent smaller receptive fields than those solitary spikes correlated with others, which may implicate that burst-like spikes could encode more detailed spatial information than solitary spikes.

\section{DISCUSSION}

In the present study, we investigated correlated activities among RGCs related to burst-like spikes and solitary spikes, and found that burst-like spikes were more frequently correlated with other neurons' activities. Further analysis suggests that correlated burst-like activities could encode more detailed spatial information of visual scenes.

\section{The significance of correlated firings among neurons}

Correlated firing activities among neurons have been extensively studied (DeVries, 1999; Shlens et al., 2008; Li et al., 2011) and it has been well accepted that concerted firings among neurons play an important role in retinal information processing. First, one of the characteristic features of the retina is that the number of retinal ganglion cells is the fewest among all kinds of neurons in the visual pathway (Barlow, 1981). The anatomy limitation makes it impossible that ganglion cells act as independent channels for visual information processing. So they might need to work together to encode visual stimulation. Multi-neuron recordings have shown that nearby ganglion cells of similar functional types have a strong tendency to fire in synchrony (Meister, 1996) and they can associate rapidly into different functional groups in response to various stimuli (Neuenschwander and Singer, 1996). The number of these dynamic groups is far beyond the number of retina ganglion cells. In this case, information transmission can be more sophisticated and efficient. Second, correlated activities of retinal neurons drive their common post-synaptic neurons in lateral geniculate nucleus (LGN) more effectively and efficiently because of spatial summation (Usrey et al., 1998; Field and Chichilnisky, 2007). Third, it is also reported that correlated activities are related to some specific functions of retina, such as color discrimination (Chen et al., 2004) and motion detection (Schwartz et al., 2007).

\section{Burst-like spikes of a neuron are strongly correlated with its neighbors' activities}

We calculated the ganglion cells' $\mathrm{Cl}$ values with a temporal resolution of $20 \mathrm{~ms}$, and found that burst-like activities of a neuron were more likely to be in correlation with spikes of its adjacent neurons.

There are three types of correlations according to the peak range in the cross-correlogram: broad (40-100 ms), medium $(10-50 \mathrm{~ms})$ and narrow (<1 ms) correlation. They were attributed respectively to shared signal from photoreceptors, shared excitation from amacrine cell via gap junction and exciting each other via gap junction (Brivanlou et al., 1998). 
A

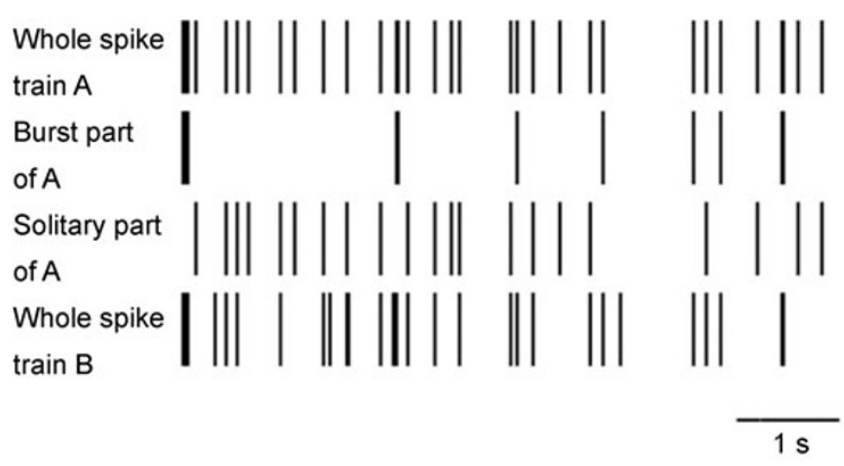

B
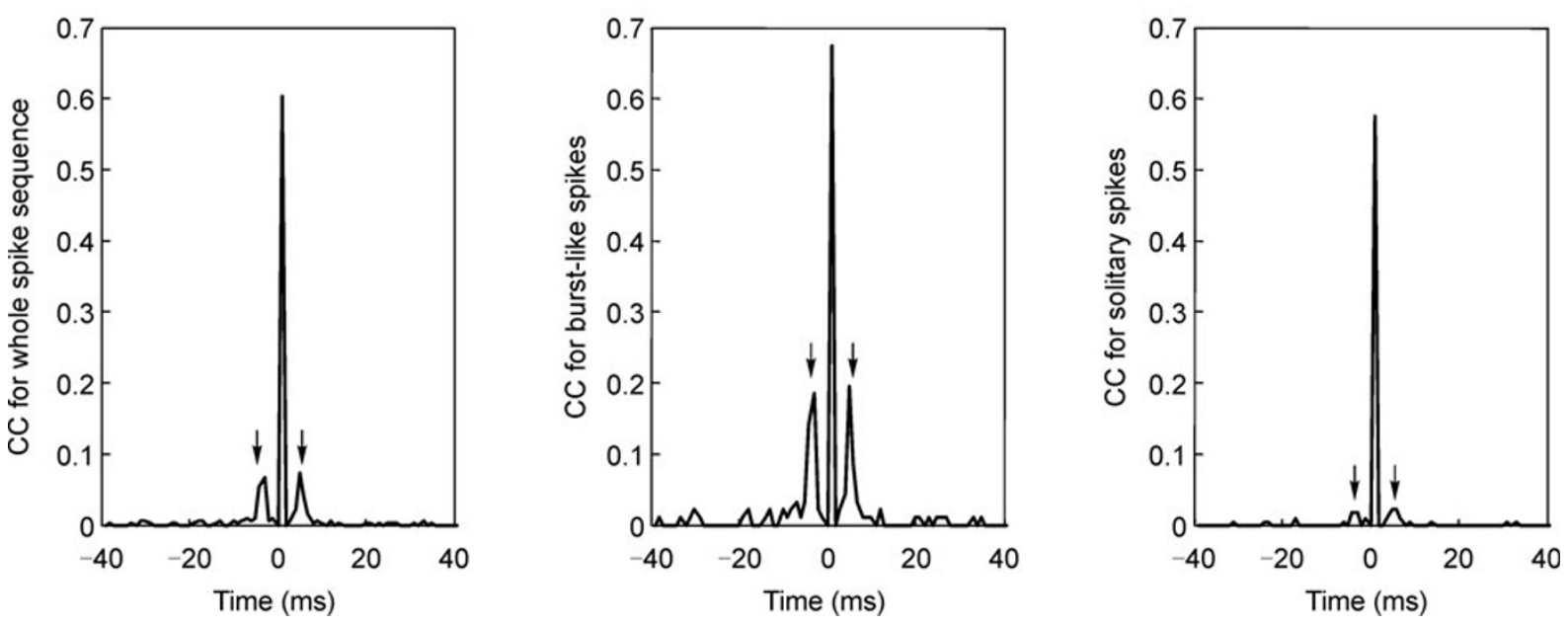

Figure 4. Cross-correlogram (CC) analysis performed on a pair of neurons. (A) Spike sequence of neuron A was separated into two parts: the burst-like spikes and the solitary spikes. (B) Cross-correlograms calculated between sequence $B$ and each part of sequence A (left: the whole sequence; middle: the burst-like spikes; right: the solitary spikes).

Given the time resolution of $20 \mathrm{~ms}$, the $\mathrm{Cl}$ value is mainly related to the correlated activity mediated by gap junctions (Brivanlou et al., 1998). Gap junctional connectivities, either among ganglion cells or between amacrine cells and ganglion cells, could result in correlated firings (Brivanlou et al., 1998; DeVries, 1999). Given that the gap junctional conductance is dependent on the voltage difference between the cells (Bloomfield and Völgyi, 2009), burst-like activities of one neuron are more likely to excite its neighboring neurons via gap junction as compared to solitary spikes (Snider et al., 1998). This may account for the result that burst-like activities had bigger $\mathrm{Cl}$ values.

\section{Physiological significance of burst-like spikes}

Correlated firings have been reported to represent smaller receptive fields (Meister et al., 1995; Schnitzer and Meister, 2003; Li et al., 2011). Our results indicate that burst-like spikes being correlated with other cells represent a smaller receptive field size, as compared to that represented by those solitary spikes being correlated with other cells, which suggests that correlated burst-like spikes help to improve spatial resolution. Although the mechanism underlying these phenomena is still not thoroughly understood, the physiological significance of burst-like spikes is quite clear.

Temporally, burst-like spikes could serve as stronger input to post-synaptic neurons, making information transmission more efficient and reliable (Lisman, 1997). Spatially, burst-like spikes are more likely to be correlated with other cells' activities (Liu et al., 2011), and correlated firing is also considered as a good way to improve the information transmission from retinal ganglion cells to their post-synaptic neurons (DeVries, 1999; Usrey et al., 1999; Sincich et al., 2007). Besides, correlated burst-like spikes represent smaller receptive fields, which contributes to detailed information encoding. These results imply that correlated burst-like spikes should be more efficient in signal transmission, and could encode more detailed spatial information. 
A
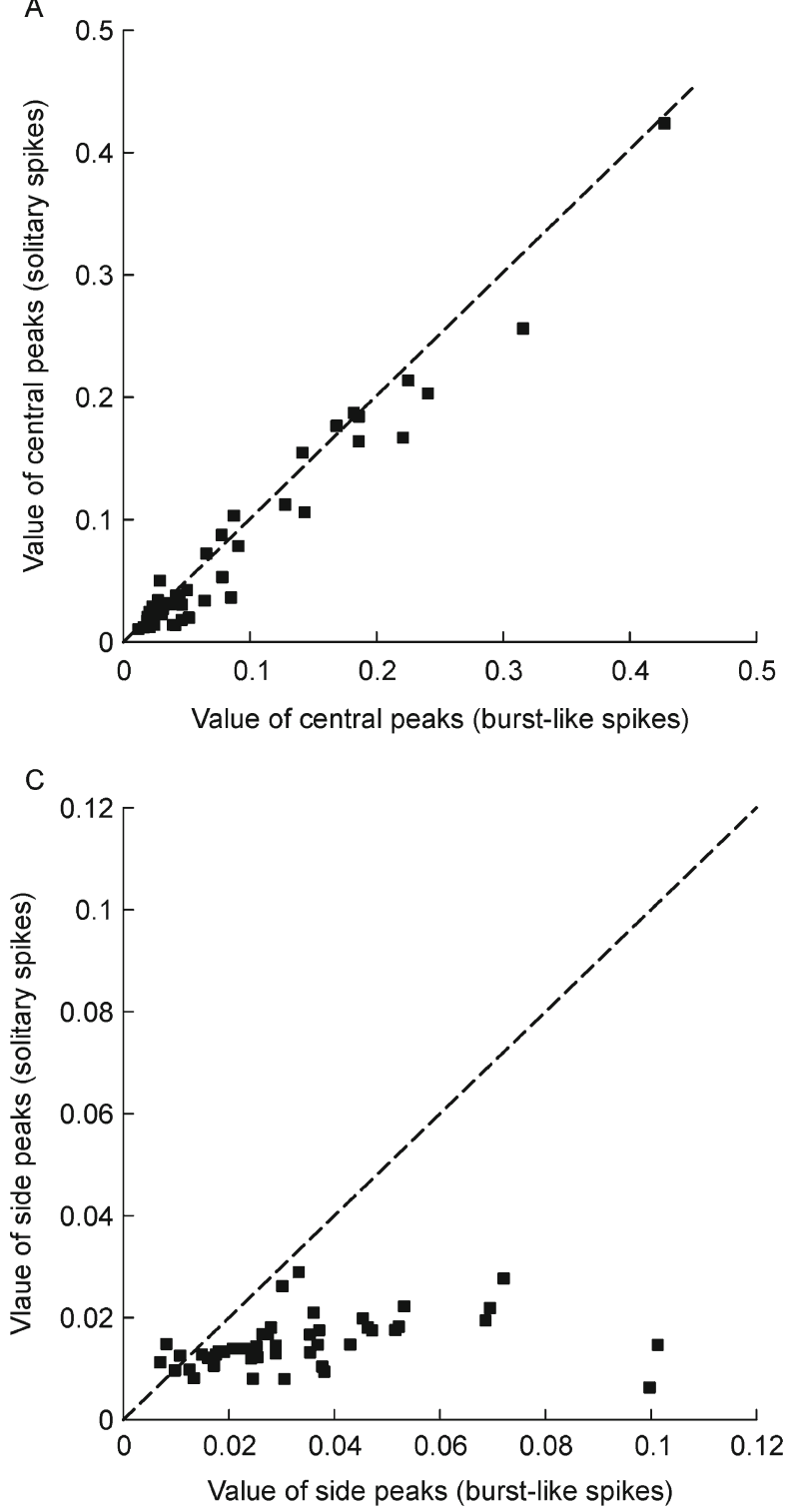

B

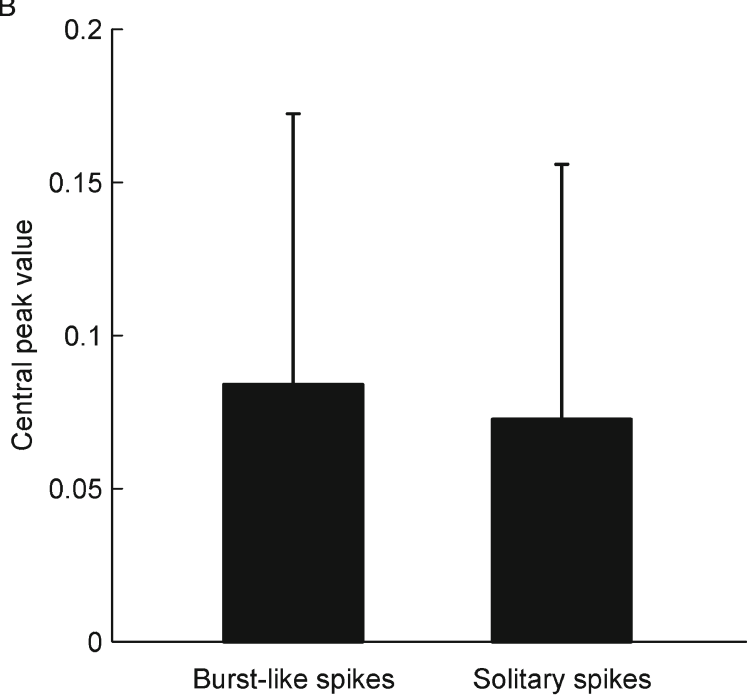

$\mathrm{D}$

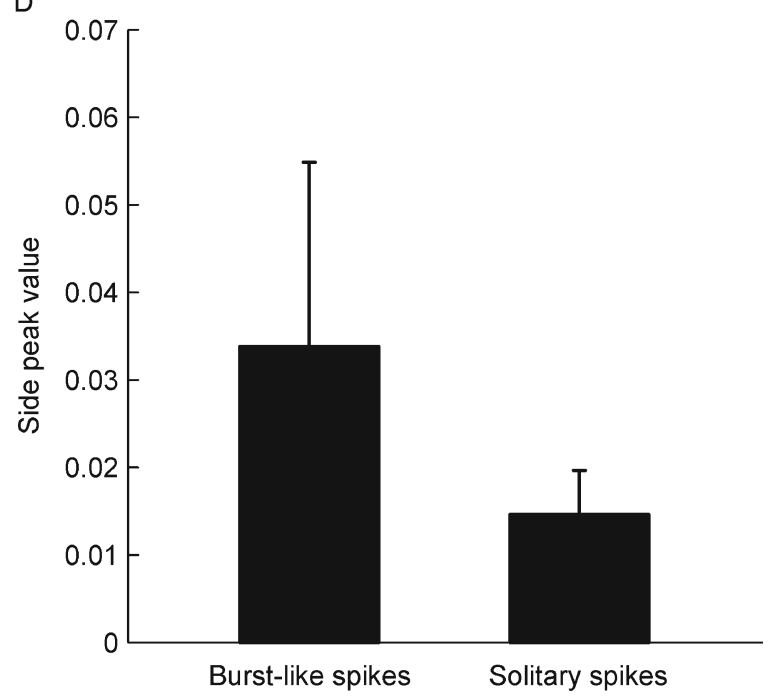

Figure 5. The comparison of central/side peaks in cross-correlogram for burst-like spikes and solitary spikes. (A) Burstlike spikes have bigger central peak values; (B) Statistics results for central peak values (mean \pm SD, burst-like spikes: $0.087 \pm 0.083$, solitary spikes: $0.072 \pm 0.084$, paired $t$-test, $n=48, p<0.05$ ); (C) Burst-like spikes have bigger side peak values; (D) Statistics results for side peak values (mean $\pm \mathrm{SD}$, burst-like: $0.034 \pm 0.021$, solitary spikes: $0.016 \pm 0.005$, paired $t$-test, $n=48, p<0.05$ ).

\section{MATERIALS AND METHODS}

\section{Experiment}

\section{Surgery}

The bullfrog was dark adapted for at least 30 minutes before experiment and then double pithed; its eyes were enucleated under dim red light. Separated eyeball was hemisected, with the eyecup being cut into small pieces, and the retina was then isolated carefully (Jing et al., 2010a). All procedures strictly conformed to the humane treatment and use of animals prescribed by the Association for Research in Vision and Ophthalmology.

\section{Electrical recording}

Multi-channel recording is a good way to study population neuronal activities (Meister et al., 1994; Jin et al., 2005; Field et al., 2007; Wang et al., 2010; Yang et al., 2010). In our experiments, neuronal activities were recorded extracellularly via MEA (MMEP-4, CNNS UNT, USA) consisted of 64 electrodes (with diameter being $8 \mu \mathrm{m}$ ) arranged in an $8 \times 8$ matrix with $150 \mu \mathrm{m}$ tip-to-tip distances between adjacent electrodes horizontally and vertically. After surgery, a small patch $(4 \mathrm{~mm} \times 4 \mathrm{~mm})$ of isolated retina was placed on MEA with the ganglion cell side contacting the electrodes. The oxygenated $\left(95 \% \mathrm{O}_{2}\right.$ and $5 \% \mathrm{CO}_{2}$ ) standard perfusate contained $100.0 \mathrm{mmol} / \mathrm{L} \mathrm{NaCl}$, 

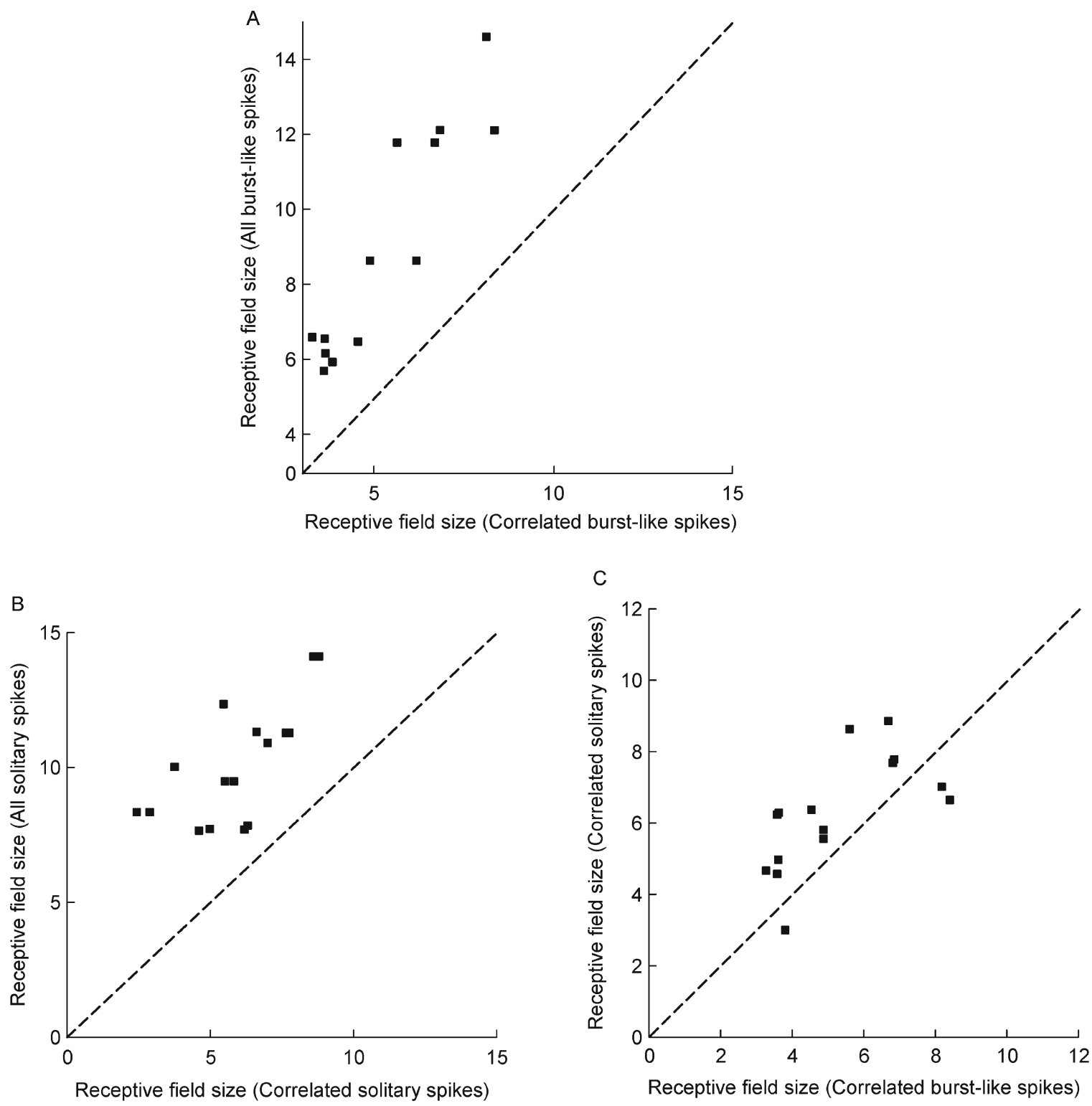

Figure 6. The comparison of receptive field size represented by different spike sequences. (A) Burst-like spikes correlated with other cells represent a smaller receptive field size when compared to all burst-like spikes; (B) solitary spikes being correlated with other cells represent a smaller receptive field size when compared to all solitary spikes; (C) correlated burst-like spikes represent a smaller receptive field size than correlated solitary spikes.

$2.5 \mathrm{mmol} / \mathrm{L} \mathrm{KCl}, 1.6 \mathrm{mmol} / \mathrm{L} \mathrm{MgCl}_{2}, 2.0 \mathrm{mmol} / \mathrm{L} \mathrm{CaCl} 2,25.0 \mathrm{mmol} / \mathrm{L}$ $\mathrm{NaHCO}_{3}$, and $10.0 \mathrm{mmol} / \mathrm{L}$ glucose.

Recorded signals were firstly amplified through a 64-channel amplifier (MEA workstation, Plexon Inc. Texas, USA) and were sampled at a rate of $40 \mathrm{kHz}$ for each channel and then stored in a Pentium-IV-based computer. Signals were high-pass filtered at $200 \mathrm{~Hz}$ to get rid of field potential. Timing signals of visual stimulus were also recorded and stored in the computer. Spike sorting was performed using the method proposed by Zhang et al. (Zhang et al., 2004), as well as the spike-sorting unit in the commercial software OfflineSorter (Plexon Inc. Texas, USA). Only single-neuron events clarified by both spike-sorting methods mentioned above were used for further analyses.

Visual stimulation

In the frog retina, RGCs can be classified into four subtypes based on their response properties: dimming detectors, sustained contrast detectors, net convexity detectors and moving-edge detectors (Lettvin et al., 1959). Light-Off stimulus evokes Off-sustained spike discharges in the dimming detectors but elicits only a few spike 
discharges transiently in other cell subtypes (Ishikane et al., 2005). Light stimulus was applied via a computer monitor (liyama, Vision Master Pro 456, Japan) and was focused to form a $1.1 \mathrm{~mm} \times 1.1 \mathrm{~mm}$ image on the isolated retina through a lens system (Jing et al., 2010a). Dimming detectors could be identified according to their light responsiveness. In the present study, only dimming detectors were selected for further analyses.

In order to study correlation characteristics of neuronal activities, pseudo-random checker-board which consisted of $16 \times 16$ subsquares was displayed on the computer monitor and refreshed at a frame rate of $20 \mathrm{~Hz}$ (Fig. 7). Each sub-square covered an area of $66 \mu \mathrm{m} \times 66 \mu \mathrm{m}$ on the retinal piece and was assigned a value either "+1" (white light, $77.7 \mathrm{nW} / \mathrm{cm}^{2}$ ) or "-1" (dark) following a pseudorandom binary sequence. Duration of the flickering stimulation and data collection was lasted for $300 \mathrm{~s}$ (Liu et al., 2011). Experiments were performed on 3 retinas.

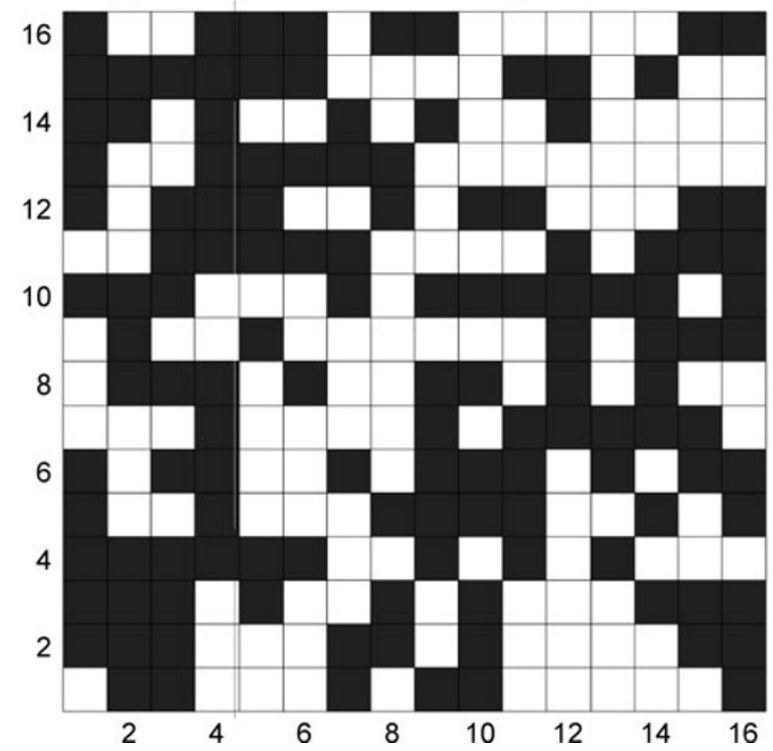

Figure 7. An example frame of checkerboard stimulation. Checker-board was consisted of $16 \times 16$ sub-squares. Each subsquare covered an area of $66 \mu \mathrm{m} \times 66 \mu \mathrm{m}$ on the retinal piece and was assigned a value either "+ 1" (white light, $77.7 \mathrm{nW} / \mathrm{cm}^{2}$ ) or "-1" (dark) following a pseudo-random binary sequence.

\section{Data analysis}

\section{Correlation Index}

For a spike train $A$, its correlation strength with another spike sequence $B$ can be calculated as follows (Liu et al., 2011). Initially both spike trains $A$ and $B$ were transformed into " $0-1$ " sequences with resolution of $20 \mathrm{~ms}$, with " 1 " indicating spike(s) in a bin and " 0 " for none. By multiplying binned sequences of $A$ and $B$, we obtained a combined sequence $A B$; Correlation strength is defined as:

$$
C l_{A B}=\frac{P_{A B}}{P_{A} P_{B}}
$$

where $P_{A B}$ is the probability of " 1 " in combined sequence $A B$, and $P_{A}$ and $P_{B}$ are the probabilities of "1" in sequences $A$ and $B$ respectively.

The $C /$ value for sequence $A$ was calculated as the mean value of all correlation strength values involving $A$ :

$$
C I_{A}=\frac{1}{M} \sum_{\mathrm{i}=1}^{M} C I_{A i}
$$

where $M$ is the number of adjacent neurons in cell $A$ 's neighboring area with a radius of $300 \mu \mathrm{m}$. This range is chosen because when the distance between two neurons is beyond $300 \mu \mathrm{m}$, their correlation strength is very weak (Liu et al., 2011).

To study correlated firing related to burst-like spikes (with $|S|<30 \mathrm{~ms}$ ) and solitary spikes (with $|S| \geqslant 30 \mathrm{~ms}$ ) respectively, each spike sequence under investigation was separated into two sub-sequences-the burst-like spike train and solitary spike train (Fig. 1), and then the correlation index was estimated for these two parts separately.

\section{Cross-correlogram}

Correlation strength between a pair of neurons was also estimated by cross-correlogram. Within a pair, spikes in one selected sequence were successively taken as reference; for each reference spike, we examined its counterpart spike train in a time window of $\pm 40 \mathrm{~ms}$, centered by the reference spiking time; we marked timing for each spike in the window. Finally all time windows were summed, generating an accumulated histogram, which indicates relationship between two examined sequences. The accumulated histogram was normalized by the number of spikes in the reference sequence.

\section{Receptive field}

The ganglion cells' receptive field properties were estimated via spike-triggered average (STA) method proposed by Meister et al (Meister et al., 1994; Jing et al., 2010b; Liu et al., 2011). The receptive field size was quantified as the number of contiguous sub-squares (arbitrary unit, a.u.) within the contour line at the level of $70 \%$ of the minimum negative value.

\section{ACKNOWLEDGEMENTS}

This work was supported by the grants from the State Key Basic Research and Development Plan (No.2005CB724301) and National Natural Science Foundation of China (Grant No.30670519).

\section{ABBREVIATIONS}

$\mathrm{AC}$, amacrine cell; $\mathrm{BC}$, bipolar cell; $\mathrm{CC}$, cross-correlogram; $\mathrm{Cl}$, correlation index; ISI, inter-spike interval; LGN, lateral geniculate nucleus; MEA, multi-electrode array; RGC, retinal ganglion cell; STA, spike triggered average

\section{REFERENCES}

Barlow, H.B. (1981). The Ferrier Lecture, 1980. Critical limiting factors in the design of the eye and visual cortex. Proc R Soc Lond B Biol Sci 212, 1-34. 
Bloomfield, S.A., and Völgyi, B. (2009). The diverse functional roles and regulation of neuronal gap junctions in the retina. Nat Rev Neurosci 10, 495-506.

Brivanlou, I.H., Warland, D.K., and Meister, M. (1998). Mechanisms of concerted firing among retinal ganglion cells. Neuron 20, 527-539.

Chen, A.H., Zhou, Y., Gong, H.Q., and Liang, P.J. (2004). Firing rates and dynamic correlated activities of ganglion cells both contribute to retinal information processing. Brain Res 1017, 13-20.

DeVries, S.H. (1999). Correlated firing in rabbit retinal ganglion cells. J Neurophysiol 81, 908-920.

Field, G., and Chichilnisky, E. (2007). Information processing in the primate retina: circuitry and coding. Annu Rev Neurosci 30, 1-30.

Field, G.D., Sher, A., Gauthier, J.L., Greschner, M., Shlens, J., Litke, A.M., and Chichilnisky, E.J. (2007). Spatial properties and functional organization of small bistratified ganglion cells in primate retina. J Neurosci 27, 13261-13272.

Hirsch, J.A., Alonso, J.M., Reid, R.C., and Martinez, L.M. (1998). Synaptic integration in striate cortical simple cells. J Neurosci 18 , 9517-9528.

Ishikane, H., Gangi, M., Honda, S., and Tachibana, M. (2005). Synchronized retinal oscillations encode essential information for escape behavior in frogs. Nat Neurosci 8, 1087-1095.

Jin, X., Chen, A.H., Gong, H.Q., and Liang, P.J. (2005). Information transmission rate changes of retinal ganglion cells during contrast adaptation. Brain Res 1055, 156-164.

Jing, W., Liu, W.Z., Gong, X.W., Gong, H.Q., and Liang, P.J. (2010a). Influence of GABAergic inhibition on concerted activity between the ganglion cells. Neuroreport 21, 797-801.

Jing, W., Liu, W.Z., Gong, X.W., Gong, H.Q., and Liang, P.J. (2010b). Visual pattern recognition based on spatio-temporal patterns of retinal ganglion cells' activities. Cogn Neurodynamics 4, 179-188.

Lettvin, J., Maturana, H., McCulloch, W., and Pitts, W. (1959). What the frog's eye tells the frog's brain. Proc IRE 47, 1940-1951.

Li, Y., Li, H., Gong, H.Q., Liang, P.J., and Zhang, P.M. (2011). Characteristics of receptive field encoded by synchronized firing pattern of ganglion cell group. Acta Biophys Sin 03, 211-221.

Lisman, J.E. (1997). Bursts as a unit of neural information: making unreliable synapses reliable. Trends Neurosci 20, 38-43.

Liu, W.Z., Jing, W., Li, H., Gong, H.Q., and Liang, P.J. (2011). Spatial and temporal correlations of spike trains in frog retinal ganglion cells. J Comput Neurosci 30, 543-553.

Meister, M. (1996). Multineuronal codes in retinal signaling. Proc Natl Acad Sci U S A 93, 609-614.

Meister, M., Lagnado, L., and Baylor, D.A. (1995). Concerted signaling by retinal ganglion cells. Science 270, 1207-1210.

Meister, M., Pine, J., and Baylor, D.A. (1994). Multi-neuronal signals from the retina: acquisition and analysis. J Neurosci Methods 51, 95-106.

Neuenschwander, S., and Singer, W. (1996). Long-range synchronization of oscillatory light responses in the cat retina and lateral geniculate nucleus. Nature 379, 728-732.

Pillow, J.W., Shlens, J., Paninski, L., Sher, A., Litke, A.M., Chichilnisky, E.J., and Simoncelli, E.P. (2008). Spatio-temporal correlations and visual signalling in a complete neuronal population. Nature 454, 995-999.

Rowe, M.H., and Fischer, Q. (2001). Dynamic properties of retinogeniculate synapses in the cat. Vis Neurosci 18, 219-231.

Schneidman, E., Berry, M.J. 2nd, Segev, R., and Bialek, W. (2006). Weak pairwise correlations imply strongly correlated network states in a neural population. Nature 440, 1007-1012.

Schnitzer, M.J., and Meister, M. (2003). Multineuronal firing patterns in the signal from eye to brain. Neuron $37,499-511$.

Schwartz, G., Taylor, S., Fisher, C., Harris, R., and Berry, M.J. 2nd. (2007). Synchronized firing among retinal ganglion cells signals motion reversal. Neuron 55, 958-969.

Shlens, J., Rieke, F., and Chichilnisky, E. (2008). Synchronized firing in the retina. Curr Opin Neurobiol 18, 396- 402.

Sincich, L.C., Adams, D.L., Economides, J.R., and Horton, J.C. (2007). Transmission of spike trains at the retinogeniculate synapse. J Neurosci 27, 2683-2692.

Snider, R.K., Kabara, J.F., Roig, B.R., and Bonds, A.B. (1998). Burst firing and modulation of functional connectivity in cat striate cortex. J Neurophysiol 80, 730-744.

Usrey, W.M., Reppas, J.B., and Reid, R.C. (1998). Paired-spike interactions and synaptic efficacy of retinal inputs to the thalamus. Nature 395, 384-387.

Usrey, W.M., Reppas, J.B., and Reid, R.C. (1999). Specificity and strength of retinogeniculate connections. J Neurophysiol 82, 3527-3540.

Wang Y., Pang J., and Lin L. (2010). Multi-channel in vivo recording in brain of freely behaving rats. Acta Biophys Sin 26, 397-405.

Yang, W., Li, Y., Li, B., and Tian, X. (2010). Time-varying spectrum synchronous pattern and behavior-event encoding by multichannel local field potentials. Acta Biophys Sin 26, 225-233.

Zhang, P.M., Wu, J.Y., Zhou, Y., Liang, P.J., and Yuan, J.Q. (2004). Spike sorting based on automatic template reconstruction with a partial solution to the overlapping problem. J Neurosci Methods $135,55-65$. 